\title{
SENSITIVITY ANALYSIS OF PWR SPENT FUEL DUE TO MODELLING PARAMETER UNCERTAINTIES USING SURROGATE MODELS
}

\author{
Bamidele Ebiwonjumi, Peng Zhang, Deokjung Lee* \\ Department of Nuclear Engineering, Ulsan National Institute of Science and Technology, 50 \\ UNIST-gil, Eonyang-eup, Ulju-gun, Ulsan, 44919, Korea
}

ebiwonjumi@unist.ac.kr, zhangpeng@unist.ac.kr, deokjung@unist.ac.kr*

\begin{abstract}
In the BEPU (Best Estimate Plus Uncertainty) framework, uncertainty quantification (UQ) is a requirement to improve confidence and reliability of code predictions. Over the years, a lot of works have been done to quantify uncertainties in code predictions of spent nuclear fuel (SNF) characteristics due to nuclear data uncertainties. The purpose of this study is to quantify the uncertainty in pressurized water reactor (PWR) fuel assembly radiation source terms (isotopic inventory, activity, decay heat, neutron and gamma source) due to uncertainties in modeling parameters. The deterministic code STREAM is used to predict the source terms of a typical PWR fuel assembly following realistic and detailed irradiation history. For the sensitivity analysis (SA) and UQ, surrogate models are developed based on polynomial chaos expansion (PCE) and variance-based global sensitivity indices (i.e., Sobol' indices) are employed. The global SA identifies the less important uncertain parameters, showing that the number of uncertain input parameters can be reduced. The surrogate model offers a significantly reduced computational burden even with large number of samples required for the SA/UQ of the model response.
\end{abstract}

KEYWORDS: STREAM, spent nuclear fuel, PWR, uncertainty quantification, surrogate models.

\section{INTRODUCTION}

The radiation source terms of spent nuclear fuel (SNF) needs to be characterized for safety applications in the back-end of the fuel cycle. SNF pools around the world are getting closer to saturation and it is difficult to measure SNF characteristics of all discharged fuels from nuclear reactors. Thus, the determination of the source terms depends largely on predictions of computer codes which model nuclide depletion during irradiation in the reactor core and decay during cooling or after discharge. The Steady state and Transient Reactor Analysis code with Method of Characteristics (STREAM) has been developed to perform light water reactor (LWR) whole core analysis [1] including support for SNF applications, deep penetration problems, radiation shielding and cask analysis. STREAM depletion and source term calculation capabilities have been validated against measurement data of SNF isotopic compositions [2] and fuel assembly (FA) decay heat [3].

Uncertainty quantification (UQ) for the calculated source terms is essential to assess the accuracy and reliability of code predictions. Over the years, several approaches have been developed to perform UQ and sensitivity analysis (SA). Examples include perturbation method and stochastic sampling. In the latter case, the inputs (nuclear data, modeling parameters) considered as uncertain are perturbed and then applied to the computer code of interest in repeated calculations. Then the response of interest is obtained

\footnotetext{
*Corresponding author
} 
and post-processed to determine the uncertainty due to the perturbed inputs. For the uncertainty due to nuclear data, covariance of nuclear data can be used.

Until now, most of the works carried out have been based on the propagation of nuclear data uncertainties on uncertainty in SNF characteristics [4-5]. Little attention has been paid to uncertainties in SNF due to uncertainties in modelling parameters [6-7] and this will be the focus of this study as it relates to pressurized water reactor (PWR) spent nuclear fuel (SNF) source terms.

The stochastic sampling approach is computationally expensive due to the large number of repeated calculations that are required. To overcome this problem, surrogate models are developed and validated, to replace STREAM. Surrogate models have become very attractive in many areas of science and engineering [8], including reactor physics SA/UQ [9-10], to replace computationally expensive code calculations. The advantages of the surrogate model are that they can be developed with small number of STREAM calculations, can be deployed with very small computational cost and can provide accurate mapping between the input data and model response. In this work, we perform forward UQ by a nonintrusive approach and a polynomial chaos expansion (PCE) based surrogate model is developed for the STREAM code during the process.

A typical PWR fuel assembly following realistic and detailed irradiation history is chosen. The SA/UQ is performed by the UQ tool UQLAB which is used to implement the PCE based surrogate model [11] and Python library Chaospy is used to calculate variance-based sensitivity indices [12]. The goal of this study is to demonstrate that PCE based surrogate models and global SA (Sobol' indices) can be employed in SNF applications to perform UQ due to modelling parameter uncertainties. The next sections of this paper are outlined as follows. The selected PWR SNF assembly is presented in Section 2 alongside the fuel design, irradiation history and uncertainty information. Section 3 describes the STREAM code, the PCE based surrogate model and global SA methods. Section 4 discusses the results and the conclusions are outlined in Section 5

\section{DESCRIPTION OF THE ASSEMBLY}

Westinghouse 15 x 15 fuel assembly design is used as the test case in this study. This assembly has been selected from a group of assemblies analyzed previously with available decay heat measurements at the Swedish central interim storage facility for spent nuclear fuel, CLAB [13]. The assembly is designated as C01, with enrichment of $3.1 \mathrm{wt}^{2}{ }^{235} \mathrm{U}$, discharge burnup of $36.7 \mathrm{GWd} / \mathrm{tU}$, and active fuel height of 365.8 $\mathrm{cm}$. The assembly contains $204 \mathrm{UO}_{2}$ fuel rods, no burnable absorber or poison rod, one instrument tube and 20 guide tubes. The assembly was irradiated in four cycles with a cooling time of about 23 years after discharge before measurement. Details of the assembly design and irradiation history information can be obtained from [13]. Uncertainties in the modeling parameters are obtained from literature and shown in Table I. The uncertainties are assumed to be uniformly distributed, and the same uncertainty information is applied to all the pins for each input parameter. The specific power is perturbed at the beginning of every cycle. All other uncertain parameters are only perturbed at the beginning of the first cycle.

\section{COMPUTATIONAL MODELS AND METHODS}

The description of the computational tools is presented in this section. The deterministic code STREAM performs the depletion and source term calculation to generate the model response. Polynomial chaos expansion based surrogate model were implemented in the UQ tool and the Python Library was used to calculate the Sobol' indices in the global SA. STREAM can be coupled to the UQ tool by a scripting interface and system call for single parameter perturbation. Uncertain parameters in STREAM are replaced by the values generated by the UQ tool. For multiple parameter perturbation, the replacement of uncertain parameters was done by post-processing. Latin Hypercube Sampling has been employed assuming a uniform distribution with mean, and standard deviation shown in Table I to generate perturbed samples. The correlations between the uncertain input parameters in Table I are not accounted for. 
Table I. Modelling parameter uncertainties (1-sigma).

\begin{tabular}{|c|c|c|}
\hline Parameter & Uncertainty & \multirow{2}{*}{ Ref. } \\
\hline Fuel density & $0.0417 \mathrm{~g} / \mathrm{cc}$ & \\
\cline { 1 - 2 }${ }^{235} \mathrm{U}$ enrichment & $0.0167 \mathrm{wt} .{ }^{235} \mathrm{U}$ & \\
\cline { 1 - 2 } Pellet radius & $0.025 \mathrm{~mm}$ & \multirow{2}{*}{ [8] } \\
\cline { 1 - 2 } Clad outer radius & $0.083 \mathrm{~mm}$ & \\
\cline { 1 - 2 } Fuel temperature & $3.33 \%$ & \\
\cline { 1 - 2 } Specific power & $1.67 \%$ & \\
\cline { 1 - 2 } Mod Temp & $2.0 \%$ & \\
\hline Boron Conc. & $2.0 \%$ & \\
\hline
\end{tabular}

\subsection{STREAM}

STREAM is an LWR analysis tool with lattice physics capabilities, neutron transport and depletion solver. STREAM uses 72-group cross sections and the pin-based slowing-down method (PSM) to generate effective multi-group cross sections. Self-shielding in the resolved resonance region is performed by solving the transport equation with collision probabilities for pin-cell. Neutron transport solution is based on the method of characteristics (MOC), adopting the assembly-modular ray tracing technique to discretize the ray tracing information and T-Y optimum quadrature set to discretize the polar angles. The anisotropic scattering is treated with the inflow transport corrected P0 model [14]. The Depletion equation is solved by the Chebyshev Rational Approximation Method (CRAM) and ENDF/BVII.0 nuclear data library is used in this work. A two-dimensional (2D) model with reflective boundary condition is used in the assembly calculation. STREAM depletion calculation for the assembly test case was modeled with detail power history in 67 burnup steps.

\subsection{Polynomial Chaos Expansion (PCE)}

PCE involves the expansion a given model response $Y(\xi)$ in terms of orthogonal basis functions of uncertain input parameters $\xi=\left(\xi_{1}, \xi_{2}, \ldots, \xi_{n_{u}}\right)$ as in Eq. (1)

$$
Y(\xi)=\sum_{n=0}^{\infty} a_{n} \psi_{n}(\xi)
$$

where $\{\psi(\xi)\}_{0}^{\infty}$ represents a complete set of orthogonal basis function. The uncertain input parameters are assumed to be independent. The basis function to be employed depends on the probability distribution of the uncertain parameters. Legendre and Hermite polynomials are commonly associated with uniformly and normally distributed parameters respectively. The series in Eq. (1) is infinite and is usually truncated at a certain order $q$, then the model response is approximated by Eq. (2).

$$
Y(\xi)=\sum_{n=0}^{q} a_{n} \psi_{n}(\xi)
$$

where $a_{n}$ are the expansion coefficients which can be used to determine the mean and variance of the model response. The expansion coefficients can be determined by numerical integration (e.g., Gaussian quadrature) or linear regression, among other methods. The number of model response evaluations $(\mathrm{m})$ 
required with the Gaussian quadrature is shown in Eq. (3), where $n_{u}$ is the number of uncertain parameters. Eq. (3) shows that the number of model evaluation is large for high-dimensional input parameters. In this work, the $a_{n}$ are determined by linear regression. Eq. (2) can be cast as a linear system and the number of unknowns $(N)$ is given by Eq. (4). $N$ number of model evaluations are required to solve Eq. (2) for the coefficients and this is much less even for a high-dimensional input. If the number of model evaluations available is greater than $N$, then we have an overdetermined system and the coefficients can be determined by least square minimization.

$$
\begin{gathered}
m=(q+1)^{n_{u}}, \\
N=\left(n_{u}+q\right) ! /\left(n_{u} ! q !\right),
\end{gathered}
$$

Details of the PCE method can be found in [11]. The model response evaluations refer to STREAM simulation result of the source terms.

\subsection{Global Sensitivity Analysis}

SA quantifies the output uncertainty of a simulation tool due to the sources of uncertainties in the input parameters. Local sensitivity analysis evaluates the output sensitivity caused by small input variation around certain input parameters using the partial derivative of the model. The global sensitivity analysis (GSA) accounts for the whole range of variation and statistical distribution of input parameters to determine the output uncertainty in a statistical framework. GSA in this paper is based on the decomposition of the variance suggested by Sobol [15]. The contribution of each input parameters to the variance of the output, otherwise known as first order Sobol' indices or the main effect, is given in Eq. (5). The total Sobol' indices or total effect considers the contribution of each input parameters plus the high order effects which are the interactions with other input parameters. The main and total effects are usually enough to know the significant input parameters.

$$
S_{i}=\frac{\operatorname{Var}\left[E\left[Y \mid \xi_{i}\right]\right.}{\operatorname{Var}[Y]}
$$

\section{RESULTS AND DISCUSSIONS}

The results of for the PCE, GSA, building the surrogate model and forward UQ are presented in this section. The workflow is stated as follows:

1) Identify the input parameters and their associated uncertainties as shown in Table I.

2) By LHS, generate 100 samples in which all the input parameters are perturbed simultaneously.

3) Perform 100 STREAM calculations. Each STREAM run takes about six minutes with a single core on a PC. The input-output data represents the design of experiment.

4) Use 90 results from the 100 simulations to build the PCE based surrogate model. For our 11dimensional input parameter problem, only 78 model evaluations are required for the linear regression method according to Eq. (4) for second order PCE.

5) Use 10 simulation results (not used to build the model) to validate the surrogate model.

6) Generate 1,000 samples for SA/UQ.

7) Replace STREAM with the surrogate model to evaluate the model responses for 1,000 samples.

8) Perform required statistics and GSA on the 1,000 model evaluations.

Validation results of the surrogate model are presented in Figs. $2-4$, comparing the probability density functions and source terms with those of STREAM. The good agreement in those figures indicates that the surrogate can accurately map the input space into the model response. The relative validation error of the surrogate shown in Table II is evaluated according to Eq. (6) where $\mu_{Y_{V a l}}$ is the sample mean of the validation set response, using the inputs and outputs of the validation set $\left[\xi_{\text {Val }}, y_{\text {Val }}=Y\left(\xi_{\text {Val }}\right)\right]$. The 
surrogate model is then applied to the large number of samples at a very cheap computational cost. The computational time required for STREAM to run 1,000 cases would be about 6,000 minutes (100 hours or 4 days) with single core on a desktop computer. The surrogate model evaluates the model response in a matter of seconds, reducing the computational burden by orders of magnitude.

$$
\epsilon_{V a l}=\frac{N-1}{N}\left[\frac{\sum_{i=1}^{N}\left(Y\left(\xi_{V a l}^{(i)}\right)-Y^{P C E}\left(\xi_{V a l}^{(i)}\right)\right)^{2}}{\sum_{i=1}^{N}\left(Y\left(\xi_{V a l}^{(i)}\right)-\mu_{Y_{V a l}}\right)^{2}}\right],
$$

Table II. Relative validation error of surrogate model (-).

\begin{tabular}{|c|c|c|c|c|c|}
\hline & Decay heat & Activity & Neutron source & Gamma source & Burnup \\
\hline At discharge & $5.141 \mathrm{E}-05$ & $5.968 \mathrm{E}-05$ & $1.246 \mathrm{E}-04$ & $5.757 \mathrm{E}-05$ & $2.861 \mathrm{E}-06$ \\
\hline After 23 years & $3.574 \mathrm{E}-04$ & $3.375 \mathrm{E}-04$ & $1.354 \mathrm{E}-03$ & $8.770 \mathrm{E}-05$ & - \\
\hline
\end{tabular}

Fig. 1 shows the uncertainties (calculated as relative standard deviation) of the SNF characteristics at discharge and after 23.2 years due to modeling parameter uncertainties. It should be noted that the nuclide number density uncertainties come from the 100 STREAM calculations previously mentioned at the beginning of this section. The neutron source shows a large uncertainty of about $6 \%$ after 23 years, followed by an uncertainty of $2 \%, 1.5 \%$, and $1.4 \%$ in the decay heat, activity and gamma source respectively. High uncertainty of neutron source is caused by ${ }^{244} \mathrm{Cm}$ (half-life 18.1 years), a dominant neutron source in the first few decades of cooling. The Sobol' sensitivity indices are presented in Fig. 5 showing the total and first order effects. The comparison of total and first order Sobol' indices can indicate if interactions between the input parameters have non-negligible effect on the variance of the output. As shown in Fig. 5, the total and first order Sobol' indices are almost the same because the interactions between the uncertain input parameters are weak. However, significant correlations between the output were observed as can be seen in Fig. 6 when analyzing the results at discharge and for the cooling time considered in this work.
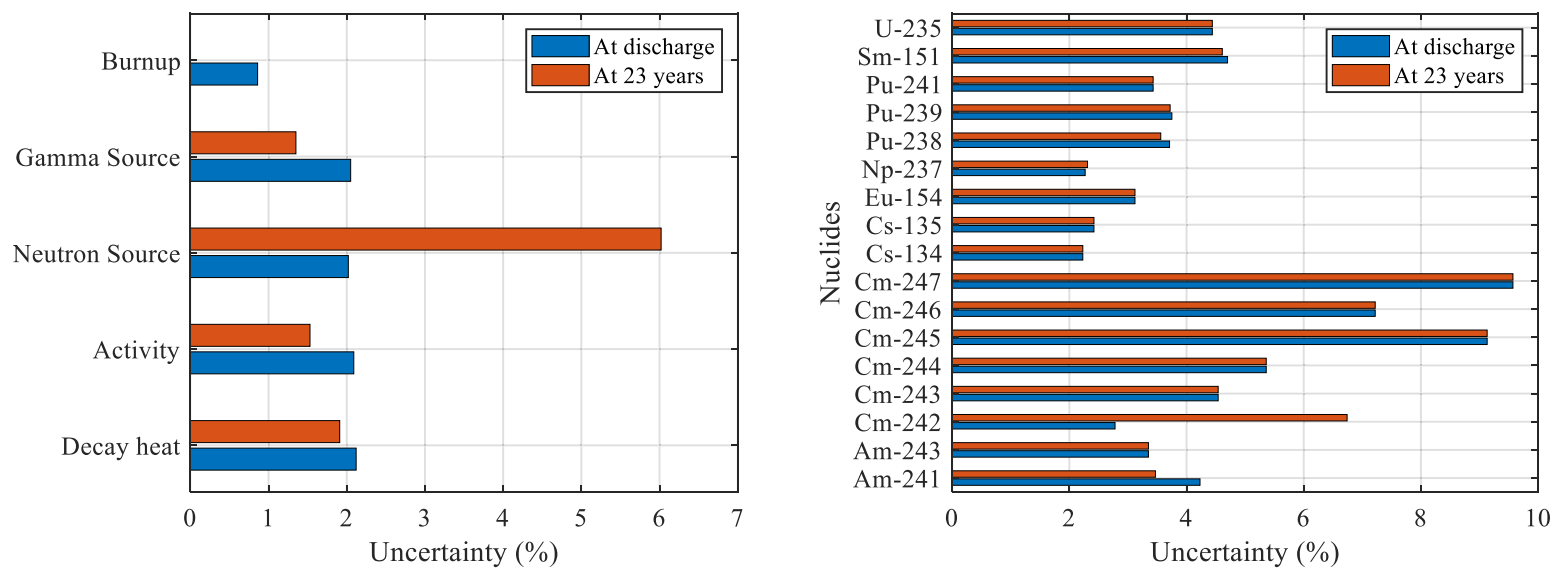

Figure 1. Uncertainties of source terms and nuclides due to modeling parameters.

To bring the results into perspective, the impact of nuclear data uncertainties in $\mathrm{PWR}^{\mathrm{U}} \mathrm{UO}_{2} \mathrm{SNF}$ is provided based on other published works. Decay heat uncertainty due to cross section reported up to 90 $\mathrm{GWd} / \mathrm{t}$ burnup. The report says the decay heat uncertainty after 100 years of cooling is less than $2 \%$ [4]. In another work considering cross sections and fission yields [5], the maximum burnup uncertainty is about $2.4 \%$ at $15 \mathrm{GWd} / \mathrm{tU}$, maximum decay heat uncertainty is $7 \%$ at $2-4$ years of cooling, maximum uncertainty in activity is $\sim 6 \%$ at $50-100$ years of cooling, maximum uncertainty in neutron source is 
$18 \%$ at $1,000-5,000$ years of cooling, and maximum uncertainty in gamma source is $\sim 9 \%$ at $2-4$ years of cooling.
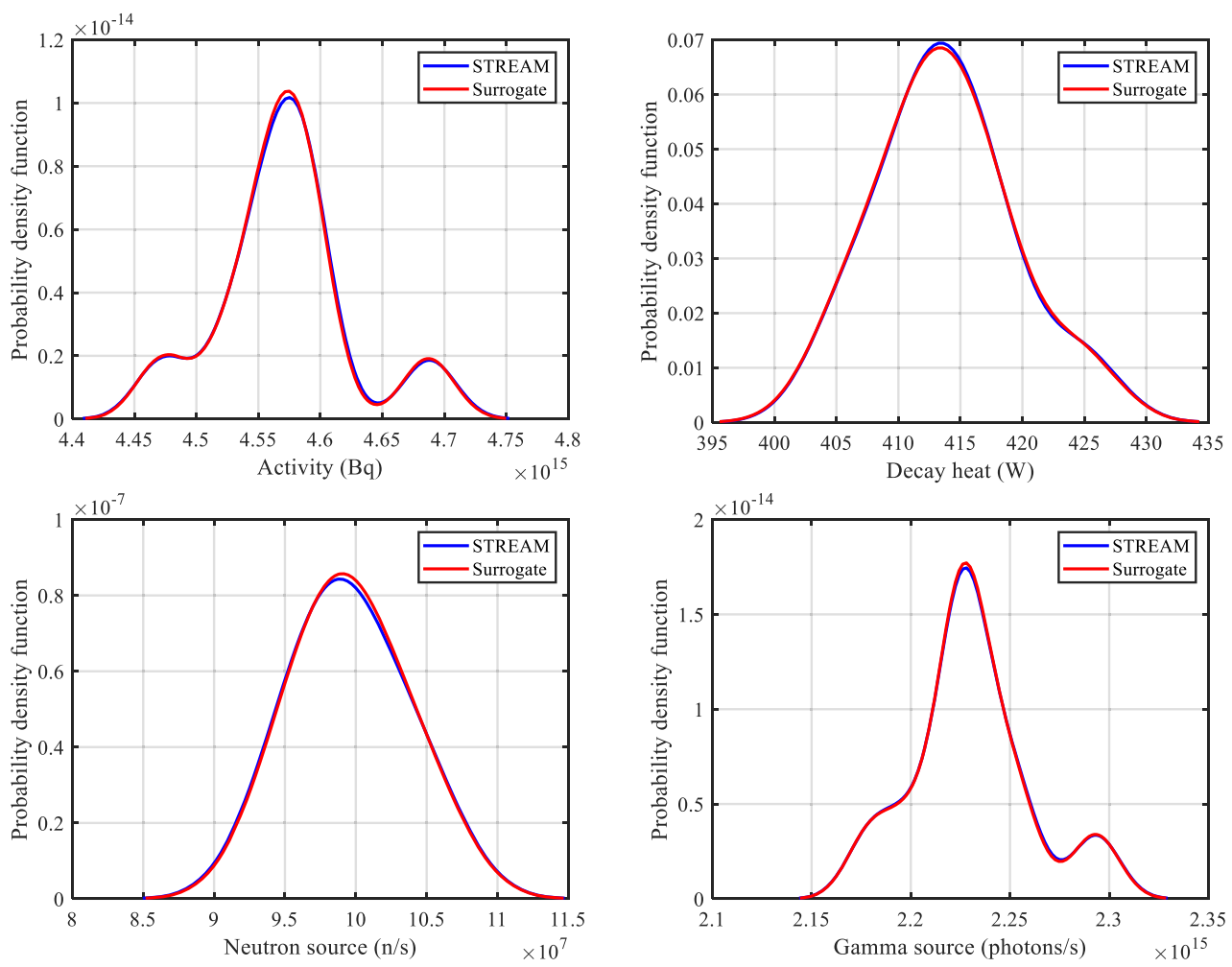

Figure 2. Comparison of PDFs at 23 years from 10 runs.
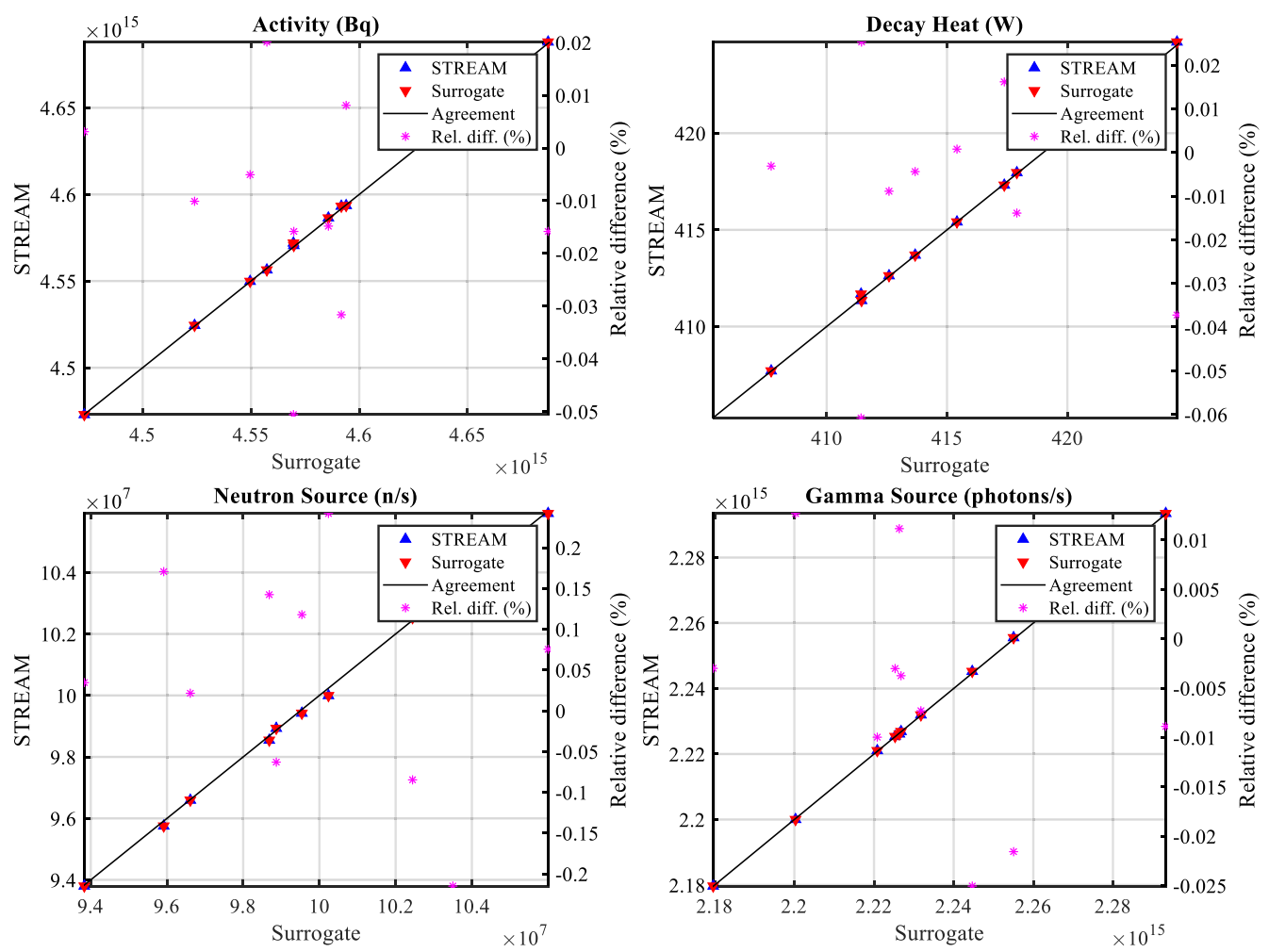

Figure 3. Comparison of source terms at 23 years from 10 runs. 

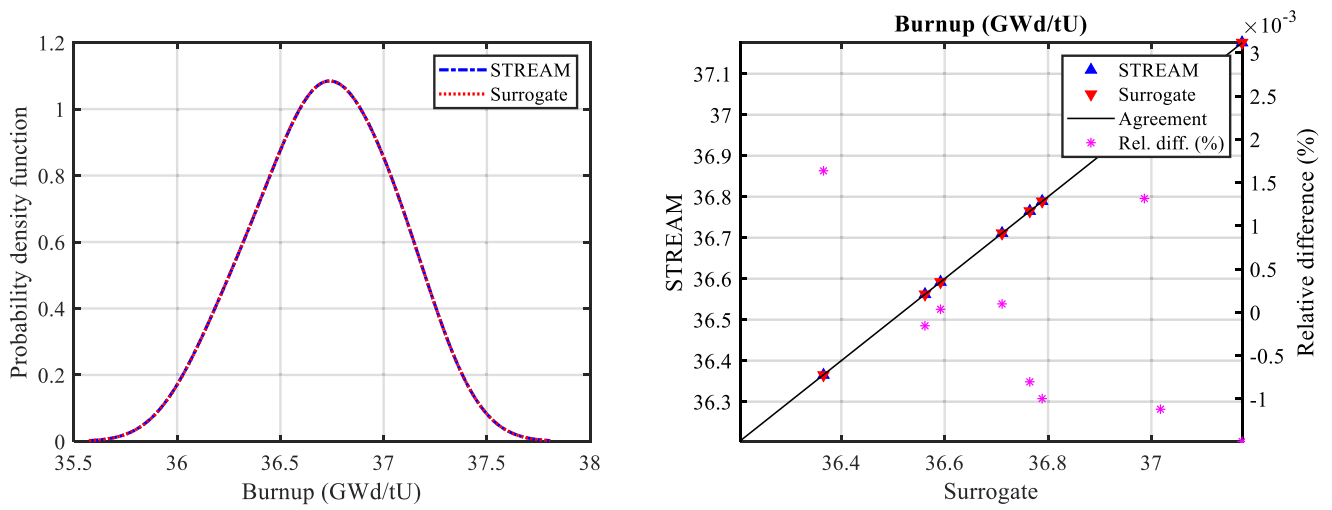

Figure 4. Comparison of burnup and it's PDFs at discharge from 10 runs.
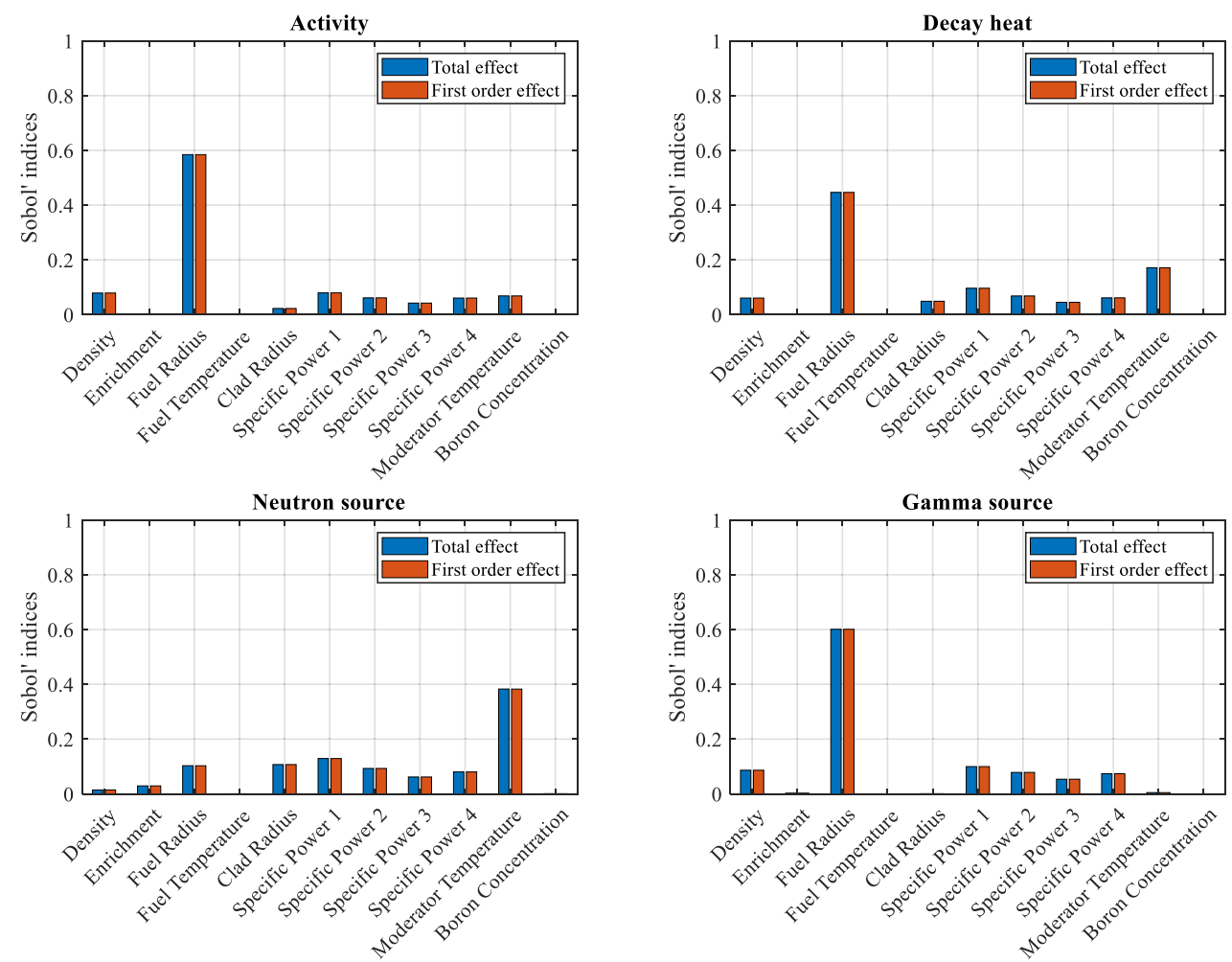

Figure 5. Sobol' indices (total and first order effects) for the 11 uncertain input parameters.
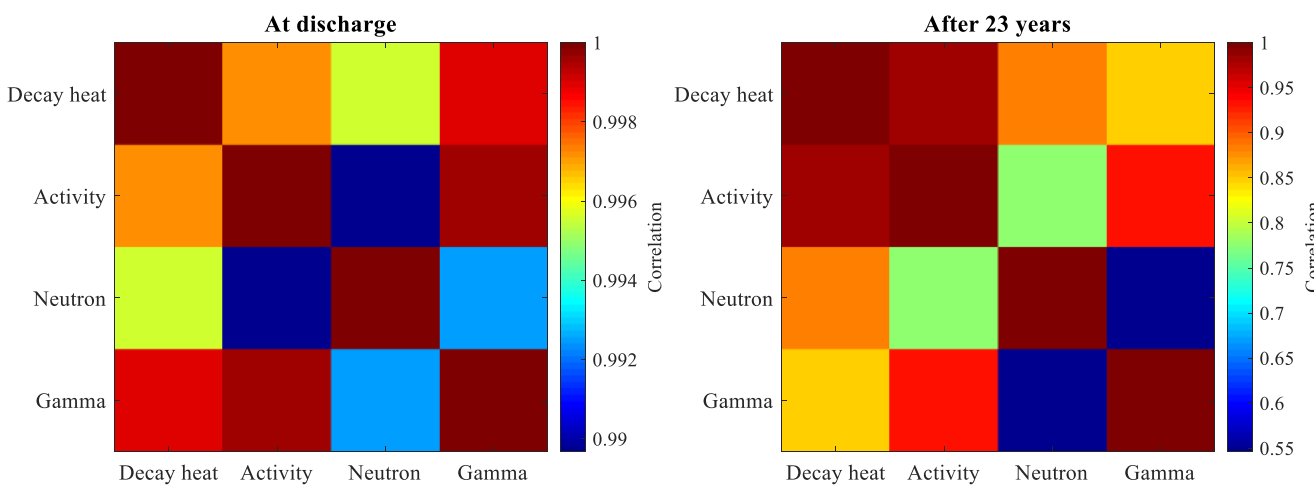

Figure 6. Correlation matrix of the source terms for the $15 \times 15$ fuel assembly. 


\section{CONCLUSIONS}

Surrogate models are applied to perform SA/UQ of SNF due to modelling parameter uncertainties. PCE based surrogate model and Sobol' indices are employed in an 11-dimensional input parameter problem, achieving significantly reduced computation time for large number of samples. Uncertainties in nuclide concentrations are also presented. It is shown that the impact of fuel design and irradiation history is important (e.g. up to $6 \%$ for the neutron source). In conclusion, modeling parameters have significant impact on SNF isotopic inventory and radiation source terms.

\section{ACKNOWLEDGMENTS}

This research was partially supported by the project(L18S015000) by Korea Hydro \& Nuclear Power Co. Ltd.. This work was partially supported by KOREA HYDRO \& NUCLEAR POWER CO., LTD (No. 2018-Tech-07)

\section{REFERENCES}

1. S. Choi, C. Lee, D. Lee, "Resonance treatment using pin-based pointwise energy slowing-down method," Journal of Computational Physics, 330, pp. 134-155 (2017).

2. B. Ebiwonjumi, S. Choi, et al., "Validation of Lattice Physics Code STREAM for Predicting Pressurized Water Reactor Spent Nuclear Fuel Isotopic Inventory," Annals of Nuclear Energy, 120, pp. 431-449 (2018).

3. B. Ebiwonjumi, S. Choi, et al., "Verification and validation of radiation source term capabilities in STREAM," Annals of Nuclear Energy, 124, pp. 80-87 (2019).

4. M. Williams, G. Ilas, et al., "A statistical sampling method for uncertainty analysis with SCALE and XSUSA,” Nucl. Technol. 183 (3), pp. 515-526 (2013).

5. D. A. Rochman, A. Vasilev, et al., "Uncertainties for Swiss LWR spent nuclear fuels due to nuclear data," EPJ Nucl. Sci. Tech., 4 (6), pp. 1-13 (2018).

6. G. Ilas, G., and H. Liljenfeldt, "Decay heat uncertainty for BWR used fuel due to modeling and nuclear data uncertainties," Nucl. Eng. Des., 319, pp. 176-184 (2017).

7. D. Rochman, A. Vasiliev, H. Ferroukhi et al., "Best Estimate Plus Uncertainty Analysis for the ${ }^{244} \mathrm{Cm}$ Prediction in Spent Fuel Characterization," Proceedings of ANS Best Estimate Plus Uncertainty International Conference (BEPU 2018), Real Collegio, Lucca, Italy, May 13 - 19, 2018.

8. K. Sargsyan, Handbook of uncertainty quantification, Chapter 19, Springer International Publishing Switzerland (2017).

9. L. Gilli, D. Lathouwers, et al., "Uncertainty quantification for criticality problems using non-intrusive and adaptive Polynomial Chaos techniques," Ann. Nucl. Energy. 56, pp. 71-80 (2013).

10. Z. Perkó, D. Lathouwers, et al., "Large scale applicability of a Fully Adaptive Non-Intrusive Spectral Projection technique: Sensitivity and uncertainty analysis of a transient," Ann. Nucl. Energy. 71, pp. 272-292 (2014).

11. S. Marelli, and B. Surety, UQLab: A framework for uncertainty quantification in Matlab, Proc. 2nd Int. Conf. on Vulnerability, Risk Analysis and Management (ICVRAM2014), Liverpool, United Kingdom, pp. 2554-2563 (2014).

12. J. Feinberg, H.P. Langtangen, "Chaospy: An open source tool for designing methods of uncertainty quantification," Journal of Computational Science, 11, pp. 45-57 (2015).

13. SKB, Measurements of decay heat in spent nuclear fuel at Swedish interim storage facility, CLAB. Svensk Kärnbränslehantering AB (SKB). Swedish Nuclear Fuel and Waste Management Co. (R-0562) (2006).

14. S. Choi, K. Smith, H.C. Lee, D. Lee, "Impact of inflow transport approximation on light water reactor analysis," Journal of Computational Physics, 299, pp. 352-373 (2015).

15. C. Prieur, Handbook of uncertainty quantification, Chapter 35, Springer International Publishing Switzerland (2017). 